\title{
A posture monitoring system with IMU for ophthalmologist while operating the slit lamp
}

\author{
Kian Sek Tee ${ }^{1}$, Eugene Low $^{2}$, Wellesly Tony ${ }^{3}$, Safinaz Binti Mohd Khialdin ${ }^{4}$, Jaysuman Bin \\ Pusppanathan $^{5}$, Chin Fhong Soon ${ }^{6}$, Toong Hai Sam ${ }^{7}$ \\ ${ }^{1,2,3}$ Faculty of Electrical and Electronic Engineering, Universiti Tun Hussein Onn Malaysia, Malaysia \\ ${ }^{4}$ Department of Ophthalmology, Pusat Kesihatan University Kebangsaan Malaysia, Malaysia \\ ${ }^{5}$ School of Biomedical Engineering \& Health Science, Universiti Teknologi Malaysia, Malaysia \\ ${ }^{6}$ Biosensor and Bioengineering Laboratory, MiNT-SRC, Universiti Tun Hussein Onn Malaysia, Malaysia \\ ${ }^{7}$ Faculty of Business, Communications and Law (FOBCAL), INTI International University, Malaysia
}

\begin{tabular}{l}
\hline Article Info \\
\hline Article history: \\
Received Jul 28, 2019 \\
Revised Oct 30, 2019 \\
Accepted Nov 13, 2019 \\
\hline Keywords: \\
IMU \\
Ophthalmologist \\
Posture \\
Posture monitoring system \\
Slit Lamp \\
Spinal cord
\end{tabular}

\begin{abstract}
The posture monitoring system monitors the posture changes of the upper body and the spinal cord applying to ophthalmologists while operating the slit lamp. The motivation to the pursuit of this system is to relate posture changes while in a static sitting posture to the spinal deviation from the g-line. Often times, the spinal deviation from the g-line is classified as bad posture because of the shift in weight distribution on the spine causing pain and discomfort in certain areas of the spine. This paper presents the design concept, the development process and the working principle of the posture monitoring system using Inertial Measurement Unit (IMU) sensor. The raw measurement data obtained from human trials is tabulated and converted into a graphical representation in which all postures can be identified and distinguished by referring the relative wave pattern. The procedure is important in the improvement on the posture monitoring system for the human trials on the ophthalmologist in future.
\end{abstract}

Copyright (C) 2020 Institute of Advanced Engineering and Science. All rights reserved.

\section{Corresponding Author:}

Kian Sek Tee,

Faculty of Electrical and Electronic Engineering,

Universiti Tun Hussein Onn Malaysia, Malaysia.

Email: tee@uthm.edu.my

\section{INTRODUCTION}

Ophthalmologists have a distinctively high risk for hazards of a diverse nature, mainlymusculoskeletal issues that are caused by the constant adjusting and movement of posture and prolonged pressure on the spinal cord caused by unhealthy posture [1-6]. Ophthalmologists specialize in eye and vision care similar to optometrists and opticians, only they are differ in their levels of training. An ophthalmologist is authorized to perform eye surgery and also conduct research on the causes and cures for eye diseases [7,8]. Therefore, posture placement is important especially for ophthalmologists because of the range of differences of patients they attend to during working hours. This diversity in patients forces ophthalmologists to constantly adjust their posture depending on the height of the patient and the position of the patient's eyes. According to the specialist, the control knobs of the slit lamp are positioned higher up which forcing them to sit with an extended arm in a praying-mantis-like posture which is very unnatural and very detrimental for the lower back [9-11]. This, in turn, has negative side effects such as back pain, neck pain caused by constriction of blood vessels and nerves and anatomical changes in the spine [2, 12-19]. Under the OHS Act, Section 2 (1), employers are required to hold the responsibility to ensure the health and safety of all workers at the work site [20]. More specifically, identifying hazards, conducting a written hazard assessment and implementing controls. Therefore as an ophthalmologist, one must acknowledge that the negative effects of incorrect posture are a work hazard and that this matter should not be disregarded. 
Posture monitoring system has been invented for years to detect posture, angular rate and force. This monitoring system is usually made up of a sensor, a microcontroller, a monitoring platform or software to process the input and produce proper output that represents the movements and changes in posture. Prior researches have been conducted using the same design concept but in different approaches and applications. X. Yan et.al [21] developed a real-time motion warning personal protective equipment (PPE) which consists of a warning mechanism that enables self awareness among construction workers to prevent WMSDs. C. Antonya et.al [22] present a new algorithm for the real-time representation of the human spine with IMU sensors in which the human body posture is analysed base on quadratic Bézier curves. H. Haggag et.al [23] applied the hierarchal joint system available in the Microsoft Kinect SDK for real time ergonomic assessment in rapid upper limb assessment (RULA). P. Li et.al [24] have developed a smart safety helmet using IMU and EEG sensor which able to reduce the ergonomic risks by tracking the head gestures and the brain activity of the worker. K. S. Tee et.al [25] had done a review on the ergonomic assessment methods and devices which are often used by the researchers in the field of ergonomics.

This paper presents an instrumental system to monitor the movement and changes of posture for ophthalmologists in order to help develop data that will contribute to the research to find the solution to this problem. This instrumental system to monitor the posture of ophthalmologists will also be used to analyze the negative side effects of incorrect posture placement and the repercussion from over-stressing the spinal cord.

\section{METHODOLOGY}

\subsection{System Overview}

The flow of the posture monitoring system starts with the instrumental system attached at the upper body of the study subject. After ensure all the setup has been done, the instrumental system will be switched on. Next, the sensor detects the change of the body posture by the study subject and generate raw data in which will be process by the microcontroller later. At the same time, the data generated can be inspected from the LCD display provided. The data received by the microcontroller from the sensor will be stored in the memory card. Lastly, the data from the memory card will be extracted and transferred to the personnel computer to be analyzed. The flowchart of the instrumental Posture Monitoring System is as illustrated in Figure 1.

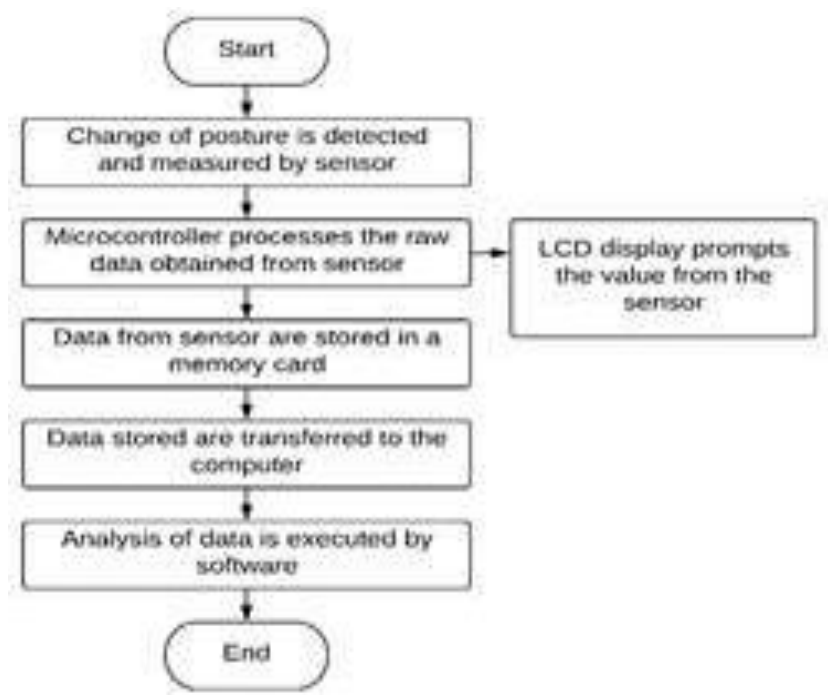

Figure 1. Flowchart of the posture monitoring system

\subsection{Design Concept}

Posture is the main point of interest in this system, the part of the body involved during sitting position should be put into consideration. The main part of the body that moves during a change in posture in sitting position is upper body-along the spine and the head. To narrow it down, the upper body is divided into three segments and the focal point of the project is the upper back (thorax).

The element of interest in this project are the parameters that become measurable variables in response to the changes of posture in ophthalmologist when working. The measurable variable when the change of posture is put into consideration is the change in angular rate in which the part of the body deviates from the 
$\mathrm{g}$-line. The change in posture is represented by the rotational movement that occurs within the 3 dimensional axis $\mathrm{x}, \mathrm{y}$, and $\mathrm{z}$. A sensor that is able to measure angular rate is required in order to provide substantial data that gives numerical value to the posture changes so that the posture change can be analysed using graphical and statistical method as well as representing the posture changes in the form of a 3D visualization of the movement using appropriate software.

The system should also be wearable and portable considering the fact that the system will be applied on the subject for a period of time. Also, it is necessary to create a wearable and portable device which is non-intrusive to the subject's natural movements. Therefore the system should be designed in a way that is wearable and ergonomic. The sensor placement will be on the upper back. This is done to allow the sensor to fully detect the changes in posture where the angle is most sensitive to the movements of the upper body. A strapping mechanism is suitable for this system as it allows for an easy wear and remove process. Figure 2 shows the design concept of the system.

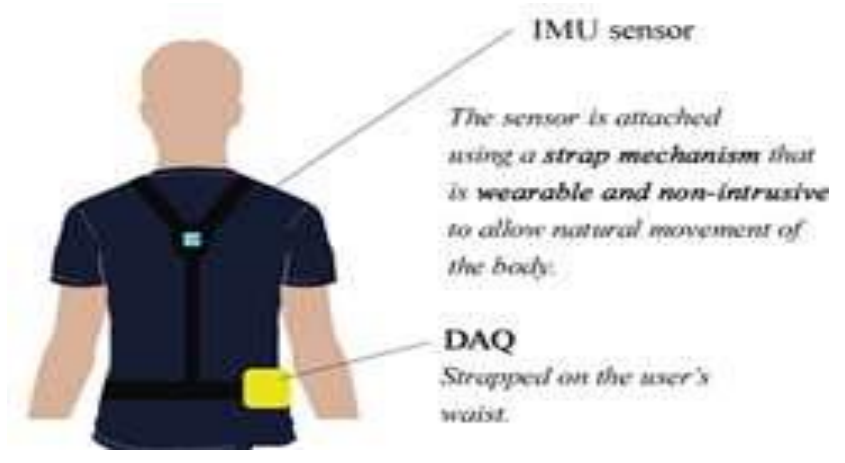

Figure 2. Design concept of the posture monitoring system

\subsection{DAQ System Design}

A circuit diagram is sketched to be used as a guideline when actual construction process for the DAQ system begins. The circuit consists of switches to start and end the running system, an SD card, variable resistors, battery, IMU sensors and LCD display. A switch will be added to control the operation of the DAQ system according to Arduino Uno. The LCD display will be connected to the digital pin of the Arduino Uno. The output voltage of the voltage divider is directed to the Arduino Uno microcontroller analogue input. The IMU sensor will be connected to the microcontroller. The IMU-GY85 connection to the Arduino is through the $\mathrm{I} 2 \mathrm{C}$ protocol. The pin for power supply depends on the IMU module itself. If the module has a $5 \mathrm{~V}$ pin, the module is connected to the $5 \mathrm{~V}$ pin. Otherwise, the module is connected to the $3.3 \mathrm{~V}$ pin on the Arduino board. The GND of the module is connected to the GND of the IMU sensor module. The SCL and SDA pins are connected between the IMU module and the Arduino board. The digital pin 2 on the Arduino board connects to the INT pin on the IMU module. All components in the system are common grounded. The block diagram of the system is shown in Figure 3.

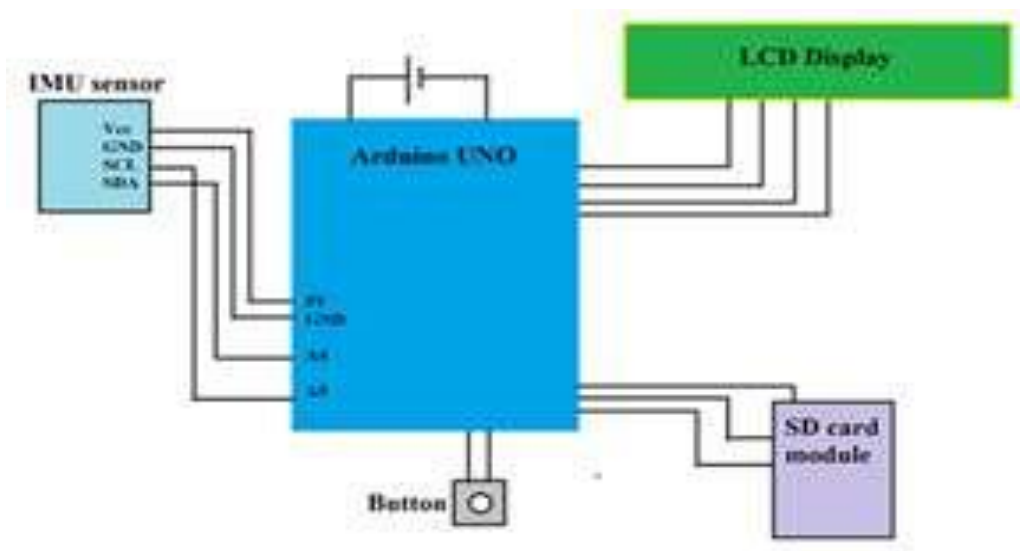

Figure 3. Block diagram of the DAQ system 


\subsection{Hardware Assembly}

All the components selected at the earlier design stage are installed in a plastic box which houses the components in place to act as the DAQ of the system. The box is then placed in a waist pack that is to be clipped around the waist to secure the position of the DAQ and allow the system to be portable. The system is powered by an external rechargeable $5 \mathrm{~V}$ battery pack. The finished product is as shown in Figure 4(a) and the circuitry of the system is as shown in Figure 4(b).

\subsection{System Validation}

System verification step helps to evaluate the functionality of the system if its meet to the design requirement. It also reduces errors during calibration process and works as planning to build the complete working system. The first verification for this system is to build an instrumental posture measurement system in which IMU sensor, Arduino UNO microcontroller, SD card and LCD display are utilized in a complete system with other minor supporting electronic components. The second verification of this system would be to verify the system functionality whether or not the system is able to measure the posture changes in the subject correctly without any errors and minimal noise and successfully save the data into the SD card. This SD card should be able to be read by the computer for further analysis.

System testing is where the system functionality is verified by running a series of test. This instrumental system will be tested by applying the system to a subject where the subject is required to wear the system for a set period of time. First, the sensor is calibrated to a point of reference where a good posture is maintained before the system starts running. Then, the subject is order to move on its own desired motion the sensor will detect and collect data within the time period to the SD card. Finally, the data from the SD card will be transferred to the computer for analysis using software. Figure 5 depicts the scenario application of the system.

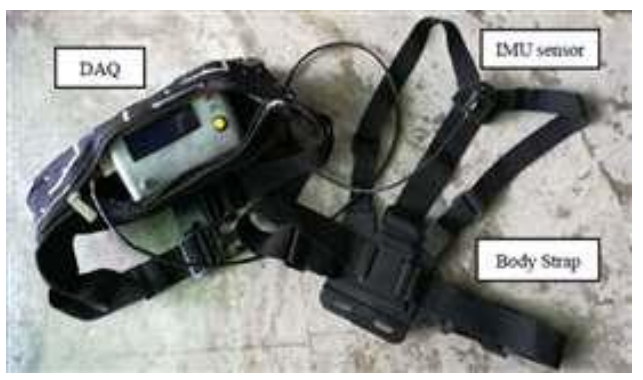

(a)

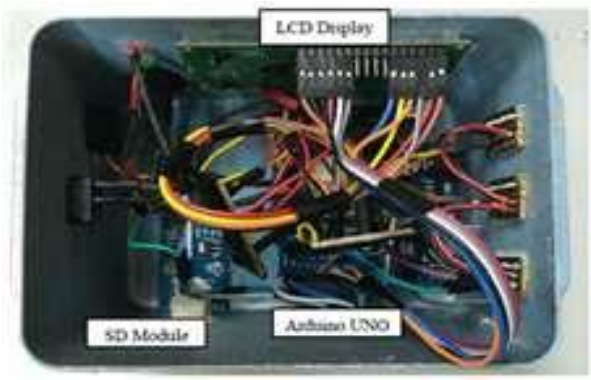

(b)

Figure 4(a). The finished product

Figure 4(b). The circuitry system

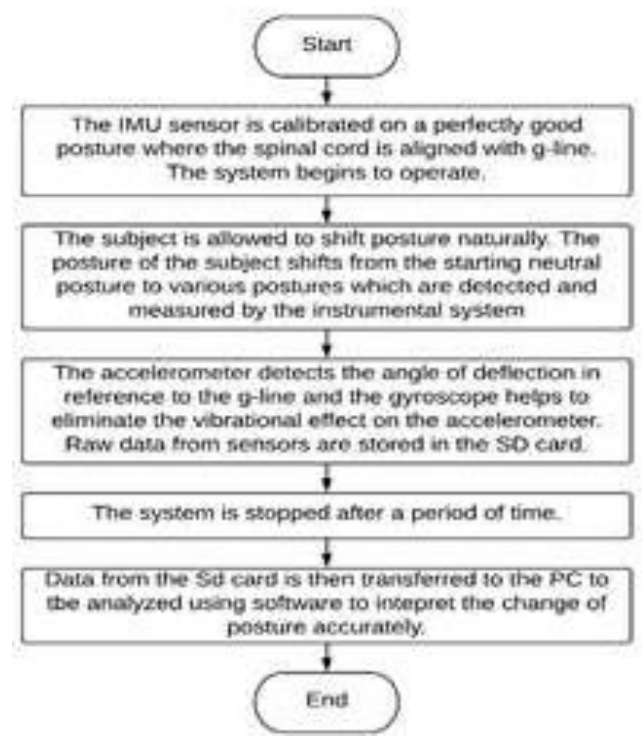

Figure 5. System testing on the posture monitoring system 


\section{RESULTS AND DISCUSSION}

The trial for the system is conducted on a human to measure the static sitting posture in various types of posture deviation to verify the functionality of the system over a specified time. The data obtained will be post-processed using Excel to obtain a graphical representation of the data collected from posture deviation in static sitting position. The first trial is for the straight or ideal posture to obtain a constant reference value and wave pattern. Table 1 is a tabulated data for the measurements obtained during the static sitting posture deviation trial for the first 20 samples in which a staright sitting posture is maintained where the spine is aligned parallel with the g-line.

Table 1. Measured data for static sitting ideal posture

\begin{tabular}{ccccccccc}
\hline Acc. X & Acc. Y & Acc. Z & Gyro. X & Gyro. Y & Gyro. Z & Mag. X & Mag. Y & Mag. Z \\
\hline 2.00. & 125 & -39 & 0 & 0.49 & 0.14 & -269 & 344 & 369 \\
3.00 & 125 & -40 & -0.49 & 0.42 & 0.42 & -265 & 340 & 370 \\
2.00 & 125 & -39 & -0.35 & 0.35 & 0.42 & -268 & 346 & 364 \\
2.00 & 126 & -39 & -0.14 & 0.35 & 0.21 & -266 & 347 & 366 \\
2.00 & 125 & -39 & -0.83 & 0.07 & 0.49 & -268 & 344 & 364 \\
3.00 & 125 & -39 & -0.42 & 0.21 & 0.35 & -268 & 345 & 364 \\
2.00 & 125 & -40 & -0.28 & 0.63 & 0.28 & -267 & 348 & 361 \\
2.00 & 126 & -38 & -0.35 & 0.42 & 0.21 & -270 & 343 & 364 \\
2.00 & 125 & -39 & -0.42 & 0.56 & 0.28 & -270 & 345 & 362 \\
2.00 & 125 & -39 & -0.7 & -0.21 & 0.28 & -268 & 345 & 365 \\
2.00 & 126 & -38 & -0.7 & 0 & 0.49 & -267 & 339 & 369 \\
2.00 & 125 & -39 & -0.49 & 0.14 & 0.77 & -269 & 341 & 366 \\
2.00 & 124 & -40 & 0 & -0.21 & 0.21 & -270 & 348 & 366 \\
2.00 & 125 & -39 & -0.77 & -0.35 & 0.49 & -270 & 338 & 366 \\
2.00 & 125 & -36 & -1.25 & -1.11 & 0.35 & -268 & 339 & 369 \\
1.00 & 126 & -39 & -0.49 & -1.18 & 0.07 & -269 & 341 & 368 \\
2.00 & 125 & -38 & -0.35 & -0.56 & 0.28 & -265 & 336 & 367 \\
1.00 & 126 & -37 & 0 & 0.14 & 0.35 & -268 & 335 & 366 \\
2.00 & 125 & -38 & -0.14 & 1.18 & 0.83 & -268 & 340 & 366 \\
2.00 & 125 & -39 & 0 & 0.49 & 0.14 & -269 & 344 & 369 \\
\hline
\end{tabular}

The sampling rate from this trial is $81 \mathrm{~Hz}$. The sampling rate is calculated by using (1).

$$
\text { Frequency }=\frac{\text { No. } \text { of samples }}{\text { Time }}
$$

The second trial is to obtain the relative wave pattern for each of the 5 distinctive static sitting postures deviations with refer to the straight posture. The 5 pre-determined static sitting postures which include straight, lean forward, lean backwards, lean right and lean left is then repeated to determine its effect in the graphical pattern of the accelerometer, magnetometer and gyroscope. Each of the five postures is repeated twice consecutively for 3 seconds where the straight position is used as the interval before transitioning to the next posture. The graphs of measurement data from the sensor are tabulated in Table 2.

For straight posture, the shape of the spine is relatively straight and perpendicular to the surface of the ground and positioned parallel with the g-line. In this posture, the spine maintains its natural curve at the thoracic region. According to the graph of the measurement data, the sensor measures a straight sitting posture where the wave pattern stays relatively constant.

In summary to the data obtained and the result findings, accelerometer $X$ responds to left and right leaning motions; accelerometer Y remain constant with sitting position; and accelerometer Z responds to forward and backward motion. As for gyroscope, gyroscope $X$ stay still with no rotation motion at yaw axis; gyroscope $Y$ create spikes on rotation motion at pitch axis for forward and backward motions; while gyroscope $\mathrm{Z}$ reacts on rotation at roll axis which falls on left and right leaning motions. As for magnetometer, only magnetometer $\mathrm{X}$ responds when it detects any changes in any position while magnetometer $\mathrm{Y}$ and $\mathrm{Z}$ are not deviating much from its original reading. 
Table 2. Graph of the measurement data of accelerometer, gyroscope and magnetometer at different postures

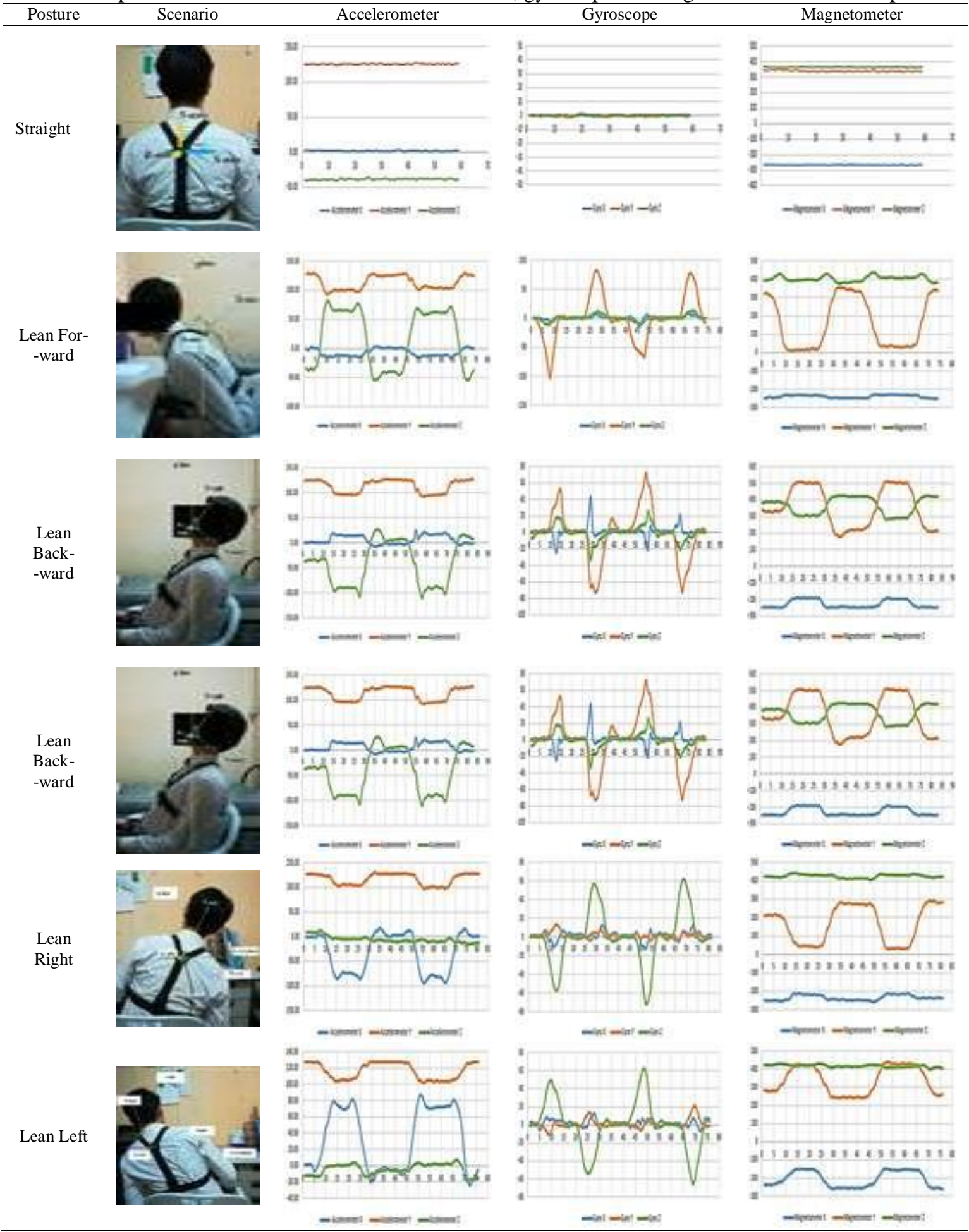

\section{CONCLUSION}

In conclusion, the proposed posture monitoring system was able to function properly and identify different postures from the study subject. The posture monitoring system can be further improved before it is ready to be applied on the ophthalmologist for human trial. In terms of mobility, the system can be further upgraded by implementing the application of the IoT to get rid of the wires from the developed system and the data can be acquired wirelessly. Furthermore, the numbers of sensors used can be increased and attached at 
different benchmark of the body parts to widen the measure area on the study subject and get more accurate data on the posture measurement.

\section{ACKNOWLEDGEMENTS}

The authors are grateful to the financial support by Postgraduate Research Grant (GPPS), Vot: U798, Universiti Tun Hussein Onn Malaysia.

\section{REFERENCES}

[1] Dhimitri, K.C., et al., "Symptoms of musculoskeletal disorders in ophthalmologists," Am J Ophthalmol, 2005. 139(1): p. $179-81$.

[2] Kitzmann, A.S., et al., "A survey study of musculoskeletal disorders among eye care physicians compared with family medicine physicians," Ophthalmology, 2012. 119(2): p. 213-20.

[3] Rambabu, T. and K. Suneetha, "Prevalence of Work Related Musculoskeletal Disorders Among Physicians," Surgeons and Dentists: A Comparative Study. Annals of Medical and Health Sciences Research, 2014. 4(4): p. 578 582.

[4] Yasobant, S. and P. Rajkumar, "Work-related musculoskeletal disorders among health care professionals: A crosssectional assessment of risk factors in a tertiary hospital," India. Indian Journal of Occupational and Environmental Medicine, 2014. 18(2): p. 75-81.

[5] Iqbal, Z. and A. Alghadir, "Prevalence of work-related musculoskeletal disorders among physical therapists," Med Pr, 2015. 66(4): p. 459-69.

[6] Wang, S.Y., et al., "Comparisons of musculoskeletal disorders among ten different medical professions in Taiwan: A nationwide, population-based study," PloS one, 2015. 10(4): p. e0123750.

[7] Society, C.O. "What is Ophthalmology? Vision Health Information from The Canadian Ophthalmological Society (COS)," 2017 [cited 2017 Jan 2017]; Available from: http://www.cos-sco.ca/vision-health-information/what-isophthalmology/

[8] Heiting, G. "How to choose an eye doctor," 2018; Available from: https://www.allaboutvision.com/eyedoctor/choose.htm

[9] Kent, C. "Will Ophthalmology Cripple You?," 2011; Available from: https://www.reviewofophthalmology.com/article/will-ophthalmology-cripple-you

[10] Chams, H., S.F. Mohammadi, and A. Moayyeri, "Frequency and assortment of self-reported occupational complaints among Iranian ophthalmologists: a preliminary survey," Medscape General Medicine, 2004. 6(4).

[11] Honavar, S.G., Head up, "heels down, posture perfect: Ergonomics for an ophthalmologist," Indian journal of ophthalmology, 2017. 65(8): p. 647.

[12] Chatterjee, A., W.G. Ryan, and E.S. Rosen, "Back pain in ophthalmologists," Eye (Lond), 1994. 8 ( Pt 4): p. 473-4.

[13] Ayanniyi, A.A., et al., "Ophthalmic practice health hazards among ophthalmologists in a resource-limited setting," Asian Pacific Journal of Tropical Disease, 2011. 1(1): p. 17-20.

[14] Hyer, J.N., et al., "National survey of back \& neck pain amongst consultant ophthalmologists in the United Kingdom," International Ophthalmology, 2015. 35(6): p. 769-775.

[15] Marx, J.L., "Ergonomics: Back to the Future," Ophthalmology, 2012. 119(2): p. 211-212.

[16] Al-Marwani Al-Juhani, M., et al., "Neck and upper back pain among eye care professionals," Occup Med (Lond), 2015. 65(9): p. 753-7.

[17] Natarajan, S. and A.G. Nair, "Is ophthalmology becoming a pain in the neck?," Indian Journal of Ophthalmology, 2016. 64(6): p. 413-414.

[18] Ratzlaff, T.D., et al., "Evaluating the efficacy of an educational ergonomics module for improving slit lamp positioning in ophthalmology residents," Canadian Journal of Ophthalmology, 2018.

[19] Venkatesh, R. and S. Kumar, " Back pain in ophthalmology: National survey of Indian ophthalmologists," Indian journal of ophthalmology, 2017. 65(8): p. 678

[20] OSHA3125, "Ergonomics: The Study of Work. 2000: U.S," Department of Labor Occupational Safety and Health Administration.

[21] Yan, X., et al., "Wearable IMU-based real-time motion warning system for construction workers' musculoskeletal disorders prevention," Automation in Construction, 2017. 74: p. 2-11.

[22] Antonya, C., S. Butnariu, and C. Pozna. "Real-time representation of the human spine with absolute orientation sensors, " in 2016 14th International Conference on Control, Automation, Robotics and Vision (ICARCV). 2016.

[23] Haggag, H., et al. "Real Time Ergonomic Assessment for Assembly Operations Using Kinect," in 2013 UKSim 15th International Conference on Computer Modelling and Simulation. 2013.

[24] Li, P., et al. "A smart safety helmet using IMU and EEG sensors for worker fatigue detection. in Robotic and Sensors Environments (ROSE)," 2014 IEEE International Symposium on. 2014. IEEE.

[25] Tee, K.S., et al., "A study on the ergonomic assessment in the workplace," AIP Conference Proceedings, 2017. 1883(1): p. 020034. 


\section{BIOGRAPHIES OF AUTHORS}
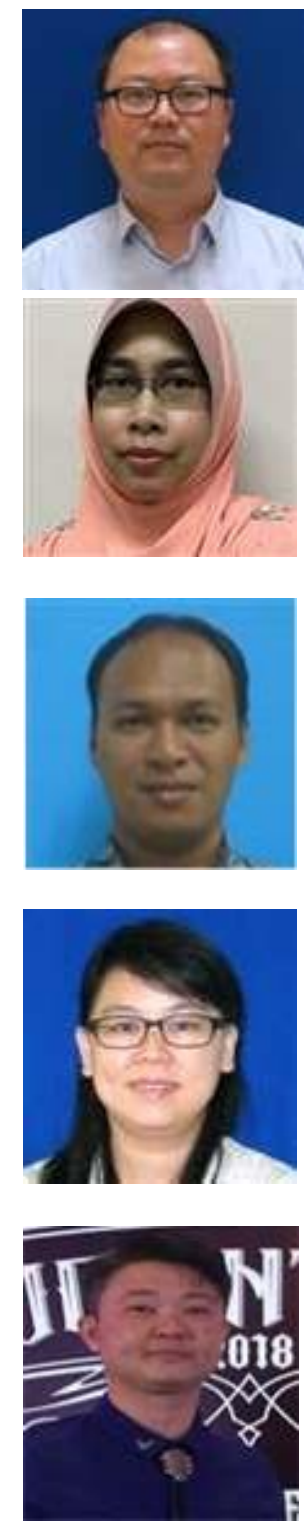

Toong Hai Samreceived the Bachelor degree in Marketing \& Management from Wichita State University, USA in 1996 and the Master degree in Business Administration from Greenwich University, Australia in 2002. He received the Ph.D. degree in Technology Management from Universiti Tun Hussein Onn Malaysia in 2018. He is currently a senior lecturer and head of program in Faculty of Business, Communications and Law, INTI International University, Malaysia. His current research interests are Biomass Renewable Energy, Agricultural Light Sports Aircraft and Unmanned Aerial Vehicles (UAV), Big Data and Narrow Band Internet of Things (IoT), E-commerce solution for Small and Medium Enterprise. 